\title{
Applying the Electronic Health Literacy Lens: Systematic Review of Electronic Health Interventions Targeted at Socially Disadvantaged Groups
}

\author{
Christina Cheng ${ }^{1,2}$, MHlthProm; Alison Beauchamp ${ }^{3,4,5}$, PhD; Gerald R Elsworth ${ }^{1}$, PhD; Richard H Osborne ${ }^{1}, \mathrm{PhD}$ \\ ${ }^{1}$ Centre for Global Health and Equity, Faculty of Health, Arts and Design, Swinburne University of Technology, Hawthorn, Australia \\ ${ }^{2}$ Deakin University, School of Health and Social Development, Faculty of Health, Burwood, Australia \\ ${ }^{3}$ Department of Rural Health, Monash University, Melbourne, Australia \\ ${ }^{4}$ Department of Medicine - Western Health, The University of Melbourne, Melbourne, Australia \\ ${ }^{5}$ Australian Institute for Musculoskeletal Science, Sunshine Hospital, St Albans, Australia
}

\section{Corresponding Author:}

Christina Cheng, MHlthProm

Centre for Global Health and Equity

Faculty of Health, Arts and Design

Swinburne University of Technology

Room 907, Level 9, AMDC Building

453/469-477 Burwood Road

Hawthorn, VIC 3122

Australia

Phone: 61392145470

Email: cccheng@swin.edu.au

\section{Abstract}

Background: Electronic health (eHealth) has the potential to improve health outcomes. However, eHealth systems need to match the eHealth literacy needs of users to be equitably adopted. Socially disadvantaged groups have lower access and skills to use technologies and are at risk of being digitally marginalized, leading to the potential widening of health disparities.

Objective: This systematic review aims to explore the role of eHealth literacy and user involvement in developing eHealth interventions targeted at socially disadvantaged groups.

Methods: A systematic search was conducted across 10 databases for eHealth interventions targeted at older adults, ethnic minority groups, low-income groups, low-literacy groups, and rural communities. The eHealth Literacy Framework was used to examine the eHealth literacy components of reviewed interventions. The results were analyzed using narrative synthesis.

Results: A total of 51 studies reporting on the results of 48 interventions were evaluated. Most studies were targeted at older adults and ethnic minorities, with only 2 studies focusing on low-literacy groups. eHealth literacy was not considered in the development of any of the studies, and no eHealth literacy assessment was conducted. User involvement in designing interventions was limited, and eHealth intervention developmental frameworks were rarely used. Strategies to assist users in engaging with technical systems were seldom included in the interventions, and accessibility features were limited. The results of the included studies also provided inconclusive evidence on the effectiveness of eHealth interventions.

Conclusions: The findings highlight that eHealth literacy is generally overlooked in developing eHealth interventions targeted at socially disadvantaged groups, whereas evidence about the effectiveness of such interventions is limited. To ensure equal access and inclusiveness in the age of eHealth, eHealth literacy of disadvantaged groups needs to be addressed to help avoid a digital divide. This will assist the realization of recent technological advancements and, importantly, improve health equity.

(J Med Internet Res 2020;22(8):e18476) doi: $\underline{10.2196 / 18476}$

\section{KEYWORDS}

eHealth; health literacy; internet; health care; telecommunications; digital divide; health equity 


\section{Introduction}

\section{Background}

Electronic health (eHealth), "the use of information and communications technology (ICT) in support of health and health-related fields" [1], is increasingly being integrated into the delivery of health resources and services. The World Health Organization (WHO) [2] also recognizes that digital technologies have the potential to accelerate toward achieving Sustainable Development Goals by improving health services. However, not everyone has substantive ICT access or skills to take advantage of the benefits of eHealth.

The issue of inequitable access, usage or skills, and outcomes relating to ICT by subgroups of society, described as the digital divide [3-6], is a recognized public health concern [7]. The sociodemographic factors associated with health disparities, such as age, income, education, and ethnicity, are similar to the characteristics of people who have limited ICT access or skills [8-10]. Older age, less education, lower income, being from an ethnic minority group, or living in a remote area are all associated with decreased access or less use of the internet for activities such as health information seeking, communicating with health care providers, monitoring health, or using personal health records [11-15]. As such, these socially disadvantaged groups are usually overlooked in eHealth design [15] and are at risk of becoming digitally marginalized $[7,16]$, leading to a potential widening of health disparities.

In recognition of the different sets of skills required for using eHealth, the concept of eHealth literacy, defined as "the ability to seek, find, understand, and appraise health information from electronic sources and apply the knowledge gained to addressing or solving a health problem," was introduced in 2006 [17]. This concept is grounded in health literacy [17,18], which is recognized as a critical determinant of health [19]. The concept of eHealth literacy has since been considered amid the everchanging landscape of ICT, and there is also a growing recognition that eHealth strategies will be ineffective and inequitable if the eHealth literacy needs of users are not addressed [20-22]. In 2015, Norgaard et al [23] developed the eHealth Literacy Framework (eHLF) by integrating the perspectives and experiences of a wide range of eHealth stakeholders, and 7 domains of eHealth literacy were identified. On the basis of this framework and applying a validity-driven approach to scale development [24], the eHealth Literacy Questionnaire (eHLQ) was also developed and tested [25]. The 7 domains of eHealth literacy are as follows: (1) using technology to process health information, (2) understanding of health concepts and language, (3) ability to actively engage with digital services, (4) feel safe and in control, (5) motivated to engage with digital services, (6) access to digital services that work and (7) digital services that suit individual needs.

According to the eHLF, eHealth literacy is not only the ability of an individual user but also relates to the system and how the two interact. For an eHealth intervention to be adopted, the system needs to align with the eHealth literacy needs of target users [23], which may differ across settings and contexts [25]. By assessing the eHealth literacy of target users, weaknesses in certain domains of eHealth literacy can be identified, and interventions can be designed to respond to the relevant weaknesses [23].

In reviewing the evaluation of the now defunct UK web-based personal health record HealthSpace, Monkman and Kushniruk [26] commented that the system did not match the eHealth literacy or information needs of users. Apart from the consideration of literacy, the evaluation also recommended that user-centered principles, such as involving users in design and development [27-29], be applied in any future endeavors [30].

\section{Objectives}

eHealth literacy plays an important role in improving health outcomes across the socioeconomic spectrum. This systematic review aimed to apply an eHealth literacy lens to explore current practices in the development of eHealth interventions targeting socially disadvantaged groups, who are at risk of being digitally marginalized. Guided by the eHLF, this review examined not only the usability of eHealth interventions but also how interventions motivate users or address privacy concerns as part of the effort to respond to eHealth literacy needs. With the WHO recognizing health literacy as having the potential to empower and drive equity [19], insights into how interventions meet the needs of disadvantaged groups will highlight gaps in research and advance the role of eHealth literacy in making eHealth more accessible. The purpose of this review was to answer the following research questions:

1. Was eHealth literacy considered during the development of eHealth interventions targeted at socially disadvantaged groups? If yes, what approaches were used to determine the eHealth literacy needs of the target group?

2. What frameworks or theories were used to guide the development of eHealth interventions besides theories on eHealth literacy?

3. Were users involved in the development of eHealth interventions?

4. What eHealth literacy domains, as described in the eHLF, were likely addressed in the identified eHealth interventions?

5. Were eHealth interventions targeted at socially disadvantaged groups effective when eHealth literacy was considered?

\section{Methods}

\section{Review Design}

This systematic review followed the Preferred Reporting Items for Systematic Reviews and Meta-Analysis Protocols 2015 checklist [31]. This was a review with no patient or public involvement.

\section{Eligibility Criteria}

The development of the inclusion criteria was based on the PICO (population, intervention, control, and outcomes) model [32]. The population referred to socially disadvantaged groups with any health condition, who were disadvantaged because of age, education, migrant status, living in a rural or remote area, or socioeconomic status [33]. For age, older adults were defined 
as people aged 60 years or older [34]. An intervention referred to eHealth interventions, systems, or applications mainly delivered through the internet via ICT such as computers, tablets, or mobile phones, targeted and operated by individual participants through platforms such as websites, apps, social media, email, or text messaging [35]. The interventions were those aimed at improving health or preventing or reducing the risk of illness. Study design included randomized controlled trials (RCTs) and non-RCT studies. Outcomes included clinical health outcomes or health knowledge and behavior. Only studies published in English peer-reviewed journals with full text available were included. Publication dates of studies were from January 2007 to July 2019. January 2007 was chosen because the concept of eHealth literacy was first introduced in late 2006 [17].

Studies were excluded if they were protocols, literature or systematic reviews, and studies of nonhealth outcomes, such as feasibility studies, usability evaluations, or economic evaluations. Studies of telehealth or telemedicine for monitoring physical conditions or medications that required no active participation from participants or only for communication with carers and health professionals were excluded. In addition, studies of consultations via videoconferencing, eHealth initiatives for risk assessment of physical conditions or motoror cognitive skills training, or computer skills training and eHealth programs targeted at health care providers or carers were excluded. In cases where studies based on the same intervention with similar outcome measures were identified, any pilot studies of that intervention were excluded.

\section{Search Strategy and Study Selection}

Studies were identified from 10 databases, including Academic Search Complete, AgeLine, Cumulative Index to Nursing and Allied Health Literature (CINAHL) Complete, Communication \& Mass Media Complete, Excerpta Medica dataBASE (EMBASE), Education Resources Information Center (ERIC), Global Health, Medical Literature Analysis and Retrieval System Online (MEDLINE) Complete, American Psychological Association PsycInfo database (PsycINFO), and Sociology Research Database (SocINDEX), with searches conducted in November 2018 and updated in July 2019. Search terms were based on keywords from the inclusion criteria (Multimedia Appendix 1). The reference lists of relevant studies were also scanned for potential studies. The search and screening of titles and abstracts were conducted by one author (CC), who also reviewed the full text of potential studies with reasons for exclusion documented.

\section{Data Extraction and Quality Assessment}

Following study selection, data were extracted based on the research questions, and study quality was appraised using the Effective Public Health Practice Project Quality Assessment
Tool (Multimedia Appendix 2) [36-87]. The tool is considered a valid and reliable instrument, adaptable to most public health systematic reviews for evaluating a range of study designs $[88,89]$. Data extraction and quality assessment of $10 \%(6 / 51)$ of the included studies were independently reviewed by 2 authors ( $\mathrm{AB}$ and $\mathrm{CC})$. Discrepancies were resolved through discussion and consensus. Decisions from the discussion were used to guide the data extraction and the quality assessment of the remaining studies undertaken by one author (CC).

\section{Data Analysis}

Owing to the heterogeneity of study designs and outcome measures among the included studies, a narrative synthesis was used to answer the research questions. For the research question relating to whether eHealth literacy domains were likely addressed in interventions, a directed content analysis approach was adopted. This approach allows researchers to use an existing theory or framework as coding categories, to develop operational definitions for each category as determined by the theory or framework, and to analyze the content accordingly [90]. For this review, the eHLF was used to code the eHealth literacy domains. The intervention components likely addressing each eHealth literacy domain were based on components derived from the concept mapping workshops used to develop the framework $[23,25]$ and matched with the description of the intervention in the included studies. For example, the use of passwords to access the system or intervention is expected to promote a sense of security. Hence, the feature is coded as meeting the needs of Domain 4 Feel safe and in control. Providing information in users' preferred language for interventions that target ethnic minorities will be a component that matches Domain 7 Digital services that suit individual needs. The classification of intervention components was initially undertaken by one author (CC), followed by discussion and review with one of the eHLF developers (RO) and among the other authors. The details of the intervention components relating to the eHealth literacy domains are presented in Table 1 [23]. The coding of $10 \%(6 / 51)$ of the studies was independently conducted by 2 authors (AB and $\mathrm{CC}$ ). Discrepancies were resolved by discussion and consensus. Decisions following discussion were used to guide the coding of the remaining studies undertaken by 1 author (CC).

For the research question regarding the effectiveness of interventions, the overall effect size could not be determined because of the diversity of outcome measures and data analysis methods. Therefore, effectiveness was estimated by reporting statistically significant improvement between intervention and control groups or between before and after intervention for one-group pretest-posttest for the outcome measures stipulated. If more than 1 primary outcome measure was stated, only the first 3 were included. 
Table 1. Examples of intervention components that likely address electronic health literacy domains derived from the eHealth Literacy Framework.

\begin{tabular}{ll}
\hline Descriptions & Examples of intervention components \\
\hline
\end{tabular}

\section{Using technology to process health information}

Able to read, write, and remember; apply basic numerical concepts; and understand context-specific language (such as health, technology, and English) as well as critically appraise information. Know when, how, and what information to use

\section{Understanding of health concepts and language}

Know about basic physiological functions and own current health status. Aware of risk factors and how to avoid them or reduce their influence on own health

\section{Ability to actively engage with digital services}

Being comfortable using digital services for handling information

\section{Feel safe and in control}

Feel that they have the ownership of personal data stored in the system and that their data are safe and can be accessed only by people to whom the data are relevant (such as own doctor and nurse)
- Contains information about health conditions

- Contains health information in a format that can easily be understood (such as text in low reading grade, video, graphics, animations, graphs, stories, examples, culturally or locally relevant materials)

- Contains information that can help make decisions

- Can use the system to share information with family, friends, and health professionals

- Can use the system to organize or record personal health information (such as recording or monitoring activities, journal, diary, worksheets)

- $\quad$ Provides access to other information resources

- Contains information that one can take responsibility for one's own health (such as setting personal goals or plans, monitoring health, practical skills or tips, practical and usable information such as recipes, activities or opportunities to join events, and download information)

- Tailored information, instructions or personal guidance, and chat sessions

- Homework assignments or tests of knowledge or evaluation

- Provide easy-to-use tools for measurements or assessment or monitoring

- Easy navigation around the system

- Detailed and easy-to-understand instructions

- $\quad$ Provide training or a manual to use the system
- Unique username and password protected

- Secure website or database or communication

- $\quad$ Provide means to ensure privacy

- Closed system to which only authorized personnel have access

- Can maintain anonymity if needed

\section{Motivated to engage with digital services}

Feel that engaging in the use of digital services will be useful for them in managing their health
- Incentives to return to use the systems

- Encouragement to continue to use the systems

- $\quad$ Alerts and notifications

- Quick response to queries

- Provides tailored feedback, progress reports, or support

- Provides new content regularly

- Regular meetup sessions or discussion forums

- Provides peer or professional support

- Quick and easy communication (such as sending or receiving emails, asking questions, and inquiries)

\section{Access to digital services that work}

Have access to digital services that the users trust to be working when they need it and as they expect it to work
- Provides access to the hardware or system

- Provides technical support

- Can be accessed anytime anywhere

- Access to tools or devices that can be integrated into the system

\section{Digital services that suit individual needs}

Have access to digital services that suit the specific needs and preferences of the users. This includes responsive features of both the information technology and health care system as well as adaptation of devices and interfaces to be used by people with physical and mental disabilities
- Consists of accessibility features such as change of font size or audio function

- $\quad$ Easy to use, efficient, and user-friendly interface (such as large buttons and large icon)

- Available in users' preferred language 


\section{Results}

\section{Selection of Studies}

The search resulted in 2640 studies; after removing 820 duplicates and an additional 25 records identified through other sources, a total of 1845 records were screened. Following a

Figure 1. Selection process using the PRISMA Flow Diagram [31].

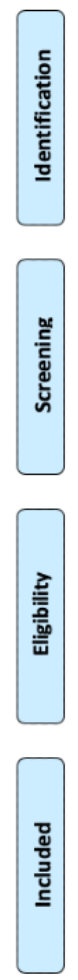

\section{Characteristics of the Studies}

Among the 51 studies, 11 were pilot studies, 43 were RCTs (40 were two-armed RCTs, 2 were three-armed RCTs, and 1 was a five-armed RCT). A total of 7 studies used the one-group pretest-posttest design, and 1 was a case study. Among the 43 RCTs, 42 control groups received no intervention, waitlist, standard care, usual care, or in-person education, whereas 1 study did not describe this. The sample size ranged from 1 to 755 (Table 2).

Apart from the postintervention assessment, 13 studies also conducted follow-up assessments, ranging from 1 week to 12 review of titles and abstracts, 75 studies were retrieved for full text review. A total of 24 studies were excluded for reasons including non-target groups, non-health outcomes, and not eHealth as defined by the inclusion criteria and 2 pilot studies of included studies, resulting in 51 studies reporting on the findings of 48 interventions (Figure 1). months. Clinical health outcomes were reported in 22 studies, whereas 28 studies measured health-related outcomes such as attitude, behaviors, or knowledge, and 1 study measured both behavior and clinical outcomes. For the quality rating, 2 studies were rated as strong, 24 studies were rated as moderate, and 25 studies were of weak quality. Among the quality rating criteria, 46 studies received a weak rating for selection bias because of their recruitment strategy or fewer than $60 \%$ of eligible participants taking part. Only 1 study received a strong rating for blinding, whereas the remaining studies either indicated blinding was not possible or did not report on blinding (Multimedia Appendix 2). 
Table 2. Characteristics of studies.

\begin{tabular}{|c|c|c|c|c|}
\hline Authors (year) & Study designs & Sample sizes & Outcome measures & Quality ratings \\
\hline Agyapong et al (2017) [37] & Two-armed RCT ${ }^{\mathrm{a}}$ & 73 & - $\quad$ BDI-II ${ }^{\mathrm{b}}$ & Strong \\
\hline Anand et al (2016) [38] & Two-armed RCT & 343 & - Myocardial Infarction Risk Score & Moderate \\
\hline Arora et al (2014) [39] & Two-armed RCT & 128 & - $\quad \mathrm{HbA}_{1 \mathrm{c}}^{\mathrm{c}}$ & Moderate \\
\hline Bennett et al (2018) [40] & Two-armed RCT & 351 & - Body weight & Weak \\
\hline Bond et al (2010) [41] & Two-armed RCT & 62 & $\begin{array}{ll}\text { - } & \text { CES-D } \\
\text { - } & \text { The Problem Areas in Diabetes Scale } \\
\text { - } & \text { Diabetes Support Scale }\end{array}$ & Weak \\
\hline $\begin{array}{l}\text { Broekhuizen et al (2016) [42] and Wijs- } \\
\text { man et al (2013) [43] }\end{array}$ & Two-armed RCT & 236 & $\begin{array}{l}\text { - Research and Development 36-item health sur- } \\
\text { vey } \\
\text { - } \quad \text { Ankle and wrist accelerometer }\end{array}$ & Moderate \\
\hline Buller et al (2008) [44] & Two-armed RCT & 755 & $\begin{array}{l}\text { - Adapted all-day screener and self-report of } \\
\text { servings }\end{array}$ & Moderate \\
\hline Carroll et al (2019) [45] & Two-armed RCT & 360 & - Patient Activation Measure & Weak \\
\hline Caster et al (2017) [46] & $\begin{array}{l}\text { One-group pretest- } \\
\text { posttest }\end{array}$ & 243 & - Knowledge scores & Weak \\
\hline Chen et al (2016) [47] & Case study & 1 & - $\quad$ Sleep satisfaction rating & Weak \\
\hline Chen et al (2018) [48] & Two-armed RCT & 233 & $\begin{array}{ll}\text { - } & \text { Attendance rate } \\
\text { - } & \text { Diabetic retinopathy } \\
\text { - } & \text { Knowledge scores }\end{array}$ & Strong \\
\hline Choi et al (2012) [49] ${ }^{\mathrm{e}}$ & Two-armed RCT & 63 & $\begin{array}{l}\text { - } \quad \text { Chinese versions of Beck Depression Inventory } \\
\text { - Chinese bilingual version of PHQ-9 }\end{array}$ & Weak \\
\hline Dang et al (2017) [50] & $\begin{array}{l}\text { Two-armed (2:1) } \\
\text { RCT }\end{array}$ & 61 & - $\quad$ Self-Efficacy for Managing Chronic Disease & Moderate \\
\hline Dear et al (2015) $[51]^{\mathrm{e}}$ & Two-armed RCT & 72 & $\begin{array}{ll}\text { - } & \text { GAD-7 } \\
\text { - } & \text { PHQ-9 }\end{array}$ & Weak \\
\hline Dugas et al (2018) [52] & Five-armed RCT & 27 & - $\quad \mathrm{HbA}_{1 \mathrm{c}}$ & Weak \\
\hline Fortmann et al (2017) [53] & Two-armed RCT & 126 & - $\quad \mathrm{HbA}_{1 \mathrm{c}}$ & Weak \\
\hline Gilmore et al (2017) [54] & Two-armed RCT & 40 & - Body weight & Moderate \\
\hline Griffin et al (2018) [55] & $\begin{array}{l}\text { One-group pretest- } \\
\text { posttest }\end{array}$ & 109 & $\begin{array}{ll}\text { - } & \text { Body weight } \\
\text { - } & \text { BMI }\end{array}$ & Weak \\
\hline Hacking et al (2016) [56] & Two-armed RCT & 223 & - Knowledge scores & Weak \\
\hline Hageman et al (2014) [57] & Three-armed RCT & 289 & $\begin{array}{ll}\text { - } & \text { Blood pressure } \\
\text { - } & \text { BMI } \\
\text { - } & \text { Waist circumference }\end{array}$ & Moderate \\
\hline Herring et al (2017) [58] & Two-armed RCT & 66 & - Body weight & Moderate \\
\hline Hill et al (2006) $[59]^{\mathrm{h}}$ & Two-armed RCT & 120 & $\begin{array}{l}\text { - } \quad \text { The Personal Resource Questionnaire } \\
\text { - } \quad \text { Rosenberg Self-Esteem Scale } \\
\text { - } \quad \text { Chronic Illness Empowerment Scale }\end{array}$ & Weak \\
\hline
\end{tabular}




\begin{tabular}{|c|c|c|c|c|}
\hline Authors (year) & Study designs & Sample sizes & Outcome measures & Quality ratings \\
\hline Hong et al (2015) [60] & $\begin{array}{l}\text { One-group pretest- } \\
\text { posttest }\end{array}$ & 30 & $\begin{array}{l}\text { - Quality of life (self-reported seven-item ques- } \\
\text { tionnaire) } \\
\text { - Level of } \mathrm{PA}^{\mathrm{i}}\end{array}$ & Weak \\
\hline Ingersoll et al (2015) [61] & Two-armed RCT & 63 & $\begin{array}{l}\text { - } \quad \text { Medication adherence (pharmacy refill data) } \\
\text { - } \quad \text { Proportion of missed visits }\end{array}$ & Moderate \\
\hline Jarvis et al (2019) [62] & Two-armed RCT & 32 & $\begin{array}{l}\text { - Disconnection and Rejection domains of the } \\
\text { Young Schema Questionnaire } \\
\text { - } \quad \text { de Jong Gierveld Loneliness Scale } \\
\text { - World Health Organization-Five Well-Being } \\
\text { Index }\end{array}$ & Weak \\
\hline Joseph et al (2015) [63] & Two-armed RCT & 29 & $\begin{array}{l}\text { - Sedentary behavior } \\
\text { - PA }\end{array}$ & Moderate \\
\hline Kamal et al (2015) [64] & Two-armed RCT & 200 & - Morisky Medication Adherence Scale & Moderate \\
\hline King et al (2013) [65] & Two-armed RCT & 40 & $\begin{array}{l}\text { - Community Health Activities Model Program } \\
\text { for Seniors questionnaire } \\
\text { - Daily steps }\end{array}$ & Moderate \\
\hline Kiropoulos et al (2011) [66] & Two-armed RCT & 202 & $\begin{array}{ll}\text { - } & \text { Depression literacy scores } \\
\text { - } & \text { Depression Stigma Scale } \\
\text { - } & \text { BDI-II }\end{array}$ & Weak \\
\hline $\begin{array}{l}\text { Lee et al (2014) [67] and Lee et al (2016) } \\
\text { [68] }\end{array}$ & $\begin{array}{l}\text { One-group pretest- } \\
\text { posttest }\end{array}$ & 30 & $\begin{array}{ll}\text { - } & \text { Adapted } 15 \text {-item scale of Taylor et al [92] } \\
\text { - } & \text { Intent (investigator developed questionnaire) } \\
\text { - } & \text { Actual vaccination or test }\end{array}$ & Weak \\
\hline Lee et al (2017) [69] & Two-armed RCT & 131 & - Completed mammograms & Weak \\
\hline MacDonell et al (2016) [70] & Two-armed RCT & 49 & $\begin{array}{l}\text { - } \quad \text { Medication adherence } \\
\text { - Asthma control }\end{array}$ & Moderate \\
\hline Marcus et al (2016) [71] & Two-armed RCT & 205 & $\begin{array}{l}\text { - Increased minutes/week of moderate to vigorous } \\
\text { PA } \\
\text { - PA by accelerometer }\end{array}$ & Moderate \\
\hline Mauriello et al (2016) [72] & Two-armed RCT & 335 & $\begin{array}{ll}\text { - } & \text { Self-reported behavior risks } \\
\text { - } & \text { Daily fruit and vegetable consumption } \\
\text { - } & \text { Daily minutes of stress management activity }\end{array}$ & Weak \\
\hline Miller et al (2018) [73] & Two-armed RCT & 450 & - Completed screening & Moderate \\
\hline Moussa et al (2013) [74] & Two-armed RCT & 45 & - Literacy Assessment for Diabetes & Moderate \\
\hline Neafsey et al (2011) [75] & Two-armed RCT & 160 & $\begin{array}{l}\text { - The Adverse Self-Medication Behavior Risk } \\
\text { Score }\end{array}$ & Moderate \\
\hline Nelson et al (2016) [76] & $\begin{array}{l}\text { One-group pretest- } \\
\text { posttest }\end{array}$ & 80 & $\begin{array}{l}\text { - Diabetes Self-Care Activities Medication sub- } \\
\text { scale }\end{array}$ & Weak \\
\hline Neuenschwander et al (2013) [77] & Two-armed RCT & 123 & $\begin{array}{l}\text { 16-item questionnaire for low-income popula- } \\
\text { tion for nutrition-related behavior outcomes }\end{array}$ & Moderate \\
\hline Phelan et al (2017) [78] & Two-armed RCT & 371 & - Body weight & Moderate \\
\hline Rubinstein et al (2016) [79] & Two-armed RCT & 637 & - Blood pressure & Moderate \\
\hline
\end{tabular}




\begin{tabular}{|c|c|c|c|c|}
\hline Authors (year) & Study designs & Sample sizes & Outcome measures & Quality ratings \\
\hline Ryan et al (2013) [80] & $\begin{array}{l}\text { One-group pretest- } \\
\text { posttest }\end{array}$ & 24 & $\begin{array}{ll}\text { - } & \mathrm{HbA}_{1 \mathrm{c}} \\
\text { - } & \text { Cholesterol }\end{array}$ & Weak \\
\hline Steinberg et al (2013) [81] & Two-armed RCT & 50 & - Body weight & Moderate \\
\hline Tessaro et al (2007) [82] & Two-armed RCT & 395 & $\begin{array}{l}\text { - } \quad \text { 34-item food frequency checklist } \\
\text { - } \quad \text { Dietary knowledge }\end{array}$ & Moderate \\
\hline Titov et al (2015) [83] ${ }^{\mathrm{e}}$ & Two-armed RCT & 54 & $\begin{array}{ll}- & \text { PHQ-9 } \\
- & \text { GAD-7 }\end{array}$ & Weak \\
\hline Ünlü Ince et al (2013) [84] & Two-armed RCT & 96 & - $\quad$ CES-D & Weak \\
\hline Wahbeh et al (2016) [85] & Two-armed RCT & 20 & $\begin{array}{ll}\text { - } & \text { CES-D } \\
\text { - } & \text { Five-Facet Mindfulness Questionnaire } \\
\text { - } & \text { Positive and Negative Affect Schedule }\end{array}$ & Moderate \\
\hline Wayne et al (2015) [86] & Two-armed RCT & 97 & - $\quad \mathrm{HbA}_{1 \mathrm{c}}$ & Weak \\
\hline Weinert et al (2008) [87] ${ }^{\mathrm{h}}$ & Three-armed RCT & 176 & $\begin{array}{l}\text { - Health knowledge score (investigator developed } \\
\text { questionnaire) }\end{array}$ & Weak \\
\hline
\end{tabular}

${ }^{\mathrm{a}} \mathrm{RCT}$ : randomized controlled trial.

${ }^{\mathrm{b}}$ BDI-II: Beck Depression Inventory II.

${ }^{\mathrm{c}} \mathrm{HbA}_{1 \mathrm{c}}$ : hemoglobin A1c.

${ }^{\mathrm{d}}$ CES-D: Center for Epidemiological Studies Depression.

eAdaptations of a similar intervention.

${ }^{\mathrm{f}}$ PHQ-9: Patient Health Questionnaire nine-item.

${ }^{g}$ GAD-7: Generalized Anxiety Disorder seven-item scale.

${ }^{\mathrm{h}}$ Same intervention but different cohorts.

${ }^{\mathrm{i}} \mathrm{PA}$ : physical activity.

\section{General Characteristics of the Interventions}

Among the 48 interventions, 32 were from the United States, 4 from Australia, 3 from Canada, 2 from the Netherlands and South Africa, and 1 each from China, Malawi, Pakistan, and Taiwan, whereas 1 intervention was undertaken across 3 South American countries, namely, Argentina, Guatemala, and Peru. Low-income groups were the most common target group $(n=20)$, followed by ethnic minorities $(n=18)$, older adults $(n=10)$, and rural communities $(n=8)$. Low-literacy groups were targeted in 2 interventions [45,73]. A wide range of health issues were addressed among the 48 interventions, with diabetes being the most common $(n=8)$, followed by 6 targeting physical inactivity and 5 targeting depression (Multimedia Appendix 3 [37-87]).

Websites were the most commonly used platforms, with 10 interventions using websites only and 12 interventions combining websites with other platforms such as email or text messaging. A total of 11 studies used text messaging alone, and 4 combined this with other platforms. A total of 10 interventions employed mobile apps. Facebook was used in 2 interventions, and WhatsApp was used in 1 intervention. Mobile phones were the most popular device, being used in 26 interventions, followed by the computer in 22 interventions. Tablets were used in 6 interventions.
Among the 48 interventions, 37 were interactive, providing information, tailored content, and/or health-engaging activities, and 11 were noninteractive, providing information or reminder text messages only. The duration of interventions ranged from one 30-min session to a 13-month program, with 3 months being the most common duration.

\section{Use of eHealth Literacy}

No interventions explicitly reported that eHealth literacy needs were considered during the development, and no assessment of eHealth literacy was undertaken. In fact, eHealth literacy was only mentioned in a study by Carroll et al [45], which included eHealth literacy as one of the secondary outcome measures and used the eHealth Literacy Scale [93] for assessment. Apart from eHealth literacy, 4 interventions undertook other literacy assessments. Ingersoll et al assessed functional English literacy by using the Wide Range Achievement Test 4 [61], and health literacy was assessed in 3 interventions using different measures, including the Short Test of Functional Health Literacy in Adults [71], the Rapid Estimate of Adults Literacy in Medicine [75], or a single question [73]. All such assessments were conducted at baseline with no discussion as to whether baseline assessment played any role in intervention development (Table 3). 
Table 3. The role of electronic health literacy and users in intervention development.

\begin{tabular}{|c|c|c|}
\hline Authors (year) & Developmental frameworks & $\begin{array}{l}\text { eHealth literacy or other literacy assessment or appli- } \\
\text { cation of user-centered principles or user involvement }\end{array}$ \\
\hline Agyapong et al (2017) [37] & - Cognitive behavioral therapy principles & - $\quad$ Content written in partnership with patients \\
\hline Anand et al (2016) [38] & $\begin{array}{l}\text { - } \quad \text { Integrative behavioral modification strategy } \\
\text { - } \quad \text { Social cognitive social learning theories } \\
\text { - } \quad \text { Goal setting theory } \\
\text { - Transtheoretical model }\end{array}$ & - $\quad$ Pilot study \\
\hline Arora et al (2014) [39] & - $\quad$ Not reported & - $\quad$ Not reported \\
\hline Bennett et al (2018) [40] & $\begin{array}{l}\text { - } \quad \text { Social cognitive theory } \\
\text { - } \quad \text { Interactive obesity treatment approach }\end{array}$ & - $\quad$ Not reported \\
\hline Bond et al (2010) [41] & - $\quad$ Not reported & - $\quad$ Not reported \\
\hline $\begin{array}{l}\text { Broekhuizen et al (2016) [42] and } \\
\text { Wijsman et al (2013) [43] }\end{array}$ & $\begin{array}{l}\text { - } \quad \text { Transtheoretical model } \\
\text { - } \quad \text { I-Change model }\end{array}$ & - $\quad$ Not reported \\
\hline Buller et al (2008) [44] & $\begin{array}{l}\text { - } \quad \text { Social cognitive theory } \\
\text { - } \quad \text { Diffusion of innovations model }\end{array}$ & $\begin{array}{ll}\text { - } & \text { Focus groups } \\
\text { - } & \text { Usability testing }\end{array}$ \\
\hline Carroll et al (2019) [45] & $\begin{array}{l}\text { - Capability, opportunity, motivation, and behavior } \\
\text { model for behavior change } \\
\text { - Community-based participatory research }\end{array}$ & $\begin{array}{l}\text { - eHealth Literacy Scale used to measure eHealth } \\
\text { literacy as one of the secondary outcomes } \\
\text { - } \quad \text { Participatory research involving users }\end{array}$ \\
\hline Caster et al (2017) [46] & - $\quad$ Not reported & - $\quad$ Focus groups \\
\hline Chen et al (2016) [47] & - $\quad$ Not reported & - $\quad$ Not reported \\
\hline Choi et al (2012) [49] $]^{\mathrm{a}}$ & $\begin{array}{l}\text { - Adaptation of the sadness internet-delivered cogni- } \\
\text { tive behavioral therapy program }\end{array}$ & - Not reported \\
\hline Dang et al (2017) [50] & - $\quad$ Not reported & - $\quad$ Not reported \\
\hline Dear et al (2015) $[51]^{\mathrm{a}}$ & - $\quad$ Previous studies & - $\quad$ Not reported \\
\hline Dugas et al (2018) [52] & - $\quad$ Not reported & - $\quad$ Not reported \\
\hline Fortmann et al (2017) [53] & - $\quad$ Not reported & - $\quad$ Not reported \\
\hline Gilmore et al (2017) [54] & $\begin{array}{l}\text { - } \quad \text { Learning theory } \\
\text { - Theory of planned behavior } \\
\text { - } \quad \text { Theory of reasoned actions } \\
\text { - Social cognitive theory }\end{array}$ & - $\quad$ Not reported \\
\hline Griffin et al (2018) [55] & - $\quad$ Social cognitive theory & - $\quad$ Not reported \\
\hline Hacking et al (2016) [56] & - Social cognitive theory & - $\quad$ Not reported \\
\hline Hageman et al (2014) [57] & $\begin{array}{l}\text { - Pender's Health Promotion Model based on social } \\
\text { cognitive theory }\end{array}$ & - Not reported \\
\hline Herring et al (2017) [58] & $\begin{array}{ll}- & \text { Social cognitive theory } \\
\text { - } & \text { Social ecological model }\end{array}$ & $\begin{array}{ll}\text { - } & \text { Focus groups } \\
\text { - } & \text { Semistructured interviews }\end{array}$ \\
\hline $\begin{array}{l}\text { Hill et al (2006) [59] and Weinert et } \\
\text { al (2008) [87] }\end{array}$ & - $\quad$ Not reported & - $\quad$ Pilot study \\
\hline
\end{tabular}




\begin{tabular}{|c|c|c|}
\hline Authors (year) & Developmental frameworks & $\begin{array}{l}\text { eHealth literacy or other literacy assessment or appli- } \\
\text { cation of user-centered principles or user involvement }\end{array}$ \\
\hline Ingersoll et al (2015) [61] & $\begin{array}{l}\text { - } \quad \mathrm{IMB}^{\mathrm{b}} \text { model of adherence } \\
\text { - }\end{array}$ & $\begin{array}{ll}\text { - } & \text { Functional English literacy assessed by Wide } \\
& \text { Range Achievement Test } 4 \\
\text { - } & \text { Focus groups } \\
\text { - } & \text { Interviews } \\
\text { - } & \text { Usability testing }\end{array}$ \\
\hline Jarvis et al (2019) [62] & $\begin{array}{l}\text { - Theoretical framework of loneliness } \\
\text { - } \quad \text { Literature review } \\
\text { Developed by a cognitive behavioral therapy spe- } \\
\text { cialist psychologist, a mental health nurse, and a } \\
\text { mobile health expert }\end{array}$ & - $\quad$ Not reported \\
\hline Joseph et al (2015) [63] & - Social cognitive theory & - $\quad$ Not reported \\
\hline Kamal et al (2015) [64] & $\begin{array}{l}\text { - } \quad \text { The health belief model } \\
\text { - Social cognitive theory } \\
\text { - } \quad \text { Michie's taxonomy of behavioral change }\end{array}$ & - $\quad$ Not reported \\
\hline King et al (2013) [65] & $\begin{array}{l}\text { - Social cognitive theory } \\
\text { - Transtheoretical model }\end{array}$ & - $\quad$ Participatory formative research \\
\hline Kiropoulos et al (2011) [66] & - $\quad$ Not reported & - $\quad$ Not reported \\
\hline $\begin{array}{l}\text { Lee et al (2014) [67] and Lee et al } \\
\text { (2016) [68] }\end{array}$ & - $\quad$ Fogg behavioral model & $\begin{array}{l}\text { - } \quad \text { Community advisory group } \\
\text { - } \quad \text { Focus groups } \\
\text { - } \quad \text { Usability testing }\end{array}$ \\
\hline Lee et al (2017) [69] & $\begin{array}{ll}\text { - } & \text { Fogg behavioral model } \\
\text { - } & \text { Health belief model } \\
\text { - } & \text { Concept of persuasive technology }\end{array}$ & $\begin{array}{l}\text { - } \quad \text { Community advisory group } \\
\text { - } \quad \text { Focus groups } \\
\text { - } \quad \text { Usability testing }\end{array}$ \\
\hline MacDonell et al (2016) [70] & $\begin{array}{l}\text { - Principles of motivational interviewing } \\
\text { - IMB skills model }\end{array}$ & - $\quad$ Pilot testing \\
\hline Marcus et al (2016) [71] & $\begin{array}{ll}\text { - } & \text { Social cognitive theory } \\
\text { - } & \text { Transtheoretical model }\end{array}$ & $\begin{array}{l}\text { - Health literacy assessed by the Short Test of } \\
\text { - Functional Health Literacy } \\
\text { - Focus groups }\end{array}$ \\
\hline Mauriello et al (2016) [72] & - Transtheoretical model of behavior change & - Usability testing \\
\hline Miller et al (2018) [73] & - $\quad$ Not reported & $\begin{array}{l}\text { - Health literacy assessed by asking a single } \\
\text { question, "how confident are you filling out } \\
\text { medical forms by yourself?" } \\
\text { - Pilot testing }\end{array}$ \\
\hline Moussa et al [74] & - $\quad$ Not reported & - $\quad$ Not reported \\
\hline Neafsey et al (2011) [75] & - Social cognitive theory & $\begin{array}{l}\text { - Health literacy assessed by Rapid Estimate of } \\
\text { - } \quad \text { Udult Literacy in Medicine } \\
\text { - } \quad \text { Pilot testing }\end{array}$ \\
\hline Nelson et al (2016) [76] & $\begin{array}{l}\text { - Adapted from the SuperEgo mobile communica- } \\
\text { tions platform }\end{array}$ & - Usability testing \\
\hline Neuenschwander et al (2013) [77] & $\begin{array}{l}\text { Kolb's learning styles and experiential learning } \\
\text { model } \\
\text { - Use of the US Department of Health and Human } \\
\text { Services' Research-based Web Design and Usabil- } \\
\text { ity Guidelines } \\
\text { - Previous users' needs and requests }\end{array}$ & - $\quad$ Pilot testing \\
\hline Phelan et al (2017) [78] & & - $\quad$ Not reported \\
\hline
\end{tabular}
(page number not for citation purposes) 


\begin{tabular}{|c|c|c|}
\hline Authors (year) & Developmental frameworks & $\begin{array}{l}\text { eHealth literacy or other literacy assessment or appli- } \\
\text { cation of user-centered principles or user involvement }\end{array}$ \\
\hline & $\begin{array}{l}\text { - Social cognitive theory } \\
\text { - Based on the diabetes prevention program and } \\
\text { Look Ahead lifestyle interventions }\end{array}$ & \\
\hline Rubinstein et al (2016) [79] & $\begin{array}{l}\text { - } \quad \text { Transtheoretical model } \\
\text { - Health belief model }\end{array}$ & $\begin{array}{ll}\text { - } & \text { Focus groups } \\
\text { - } & \text { Pilot study }\end{array}$ \\
\hline Ryan et al (2013) [80] & - Social cognitive theory & - $\quad$ Not reported \\
\hline Steinberg et al (2013) [81] & - Interactive obesity treatment approach & - $\quad$ Not reported \\
\hline Tessaro et al (2007) [82] & $\begin{array}{ll}\text { - } & \text { Health belief model } \\
-\quad & \text { Social learning theory } \\
\text { - } & \text { Social support theory }\end{array}$ & - Focus groups \\
\hline Titov et al (2015) [83] ${ }^{\mathrm{a}}$ & - $\quad$ Psychological principles & - $\quad$ Not reported \\
\hline Ünlü Ince et al (2013) [84] & - $\quad$ Not reported & - $\quad$ Not reported \\
\hline Wahbeh et al [85] & $\begin{array}{l}\text { - Modification of the mindfulness-based cognitive } \\
\text { therapy and mindfulness-based stress reduction }\end{array}$ & - $\quad$ Not reported \\
\hline Wayne et al (2015) [86] & $\begin{array}{l}\text { - } \quad \text { Motivational interviewing } \\
\text { - }\end{array}$ & - Pilot study \\
\hline
\end{tabular}

${ }^{\mathrm{a}}$ Adaptations of similar programs.

b IMB: Information, Motivation and Behavior Skills

\section{Use of Developmental Framework}

Theoretical frameworks were the most used guidelines for developing interventions, with social cognitive theories $(n=15)$ and the transtheoretical model $(n=6)$ most commonly used. A total of 7 interventions were adaptations or modifications of previous programs, whereas 13 interventions did not provide any details about their theoretical frameworks or developmental frameworks. Only 1 intervention was reported on using the Research-based Web Design and Usability Guidelines developed by the US Department of Health and Human Services (UDHHS) [94] to inform the creation of their intervention website (Table 3) [77].

\section{User Involvement}

User-centered principles were not discussed in any of the papers. The development of interventions in the included studies was mostly expert driven. A total of 22 interventions reported on involving users during development, with needs assessments using focus groups or interviews in 8 interventions, usability or pilot testing in 15 interventions, and 2 interventions mentioning participatory formative research with no details provided. Only Agyapong et al [37] reported that patients were involved in content writing (Table 3 ).

\section{Addressing eHealth Literacy Domains}

Most reviewed interventions did not address all eHealth literacy domains. The number of domains addressed ranged from 1 to 7 , with only 1 study containing components addressing all 7 eHealth literacy domains [65]. A total of 7 studies representing
6 interventions contained components of 6 domains $[57,59,64,71,78,80,87]$, whereas 20 studies contained components addressing 5 domains (Multimedia Appendix 4 [37-87]).

The 2 most addressed eHealth literacy domains, 1. Using technology to process health information and 2.Understanding of health concepts and language, were both identified in 45 interventions. The domain 5. Motivated to engage with digital services was addressed in 37 interventions through different strategies to encourage users to engage with interventions. A total of 26 interventions provided access to hardware, data plans, or technical support to address the domain 6. Access to digital services that work, whereas 23 interventions supported the domain 4. Feel safe and in control by requiring personal log-in or other forms of privacy measures. The 2 most overlooked domains were 3. Ability to actively engage with digital services and 7. Digital services that suit individual needs; both were identified in less than half of the 48 interventions. Of the 19 interventions containing components of 3. Ability to actively engage with digital services, 15 provided training or instructions on using the intervention, whereas only 6 featured an easy-to-use navigation interface. Among the 18 programs addressing the domain 7. Digital services that suit individual needs, the main strategy was to provide the preferred language of users. Accessibility features catering to individual capability or providing a user interface that suited individual needs such as large fonts or icons or audio options were only identified in 6 interventions [64,65,73-75,82]. Figure 2 shows the number of interventions addressing each of the 7 domains. 
Figure 2. Number of interventions addressing the seven domains of the eHealth Literacy Framework [23, 25].

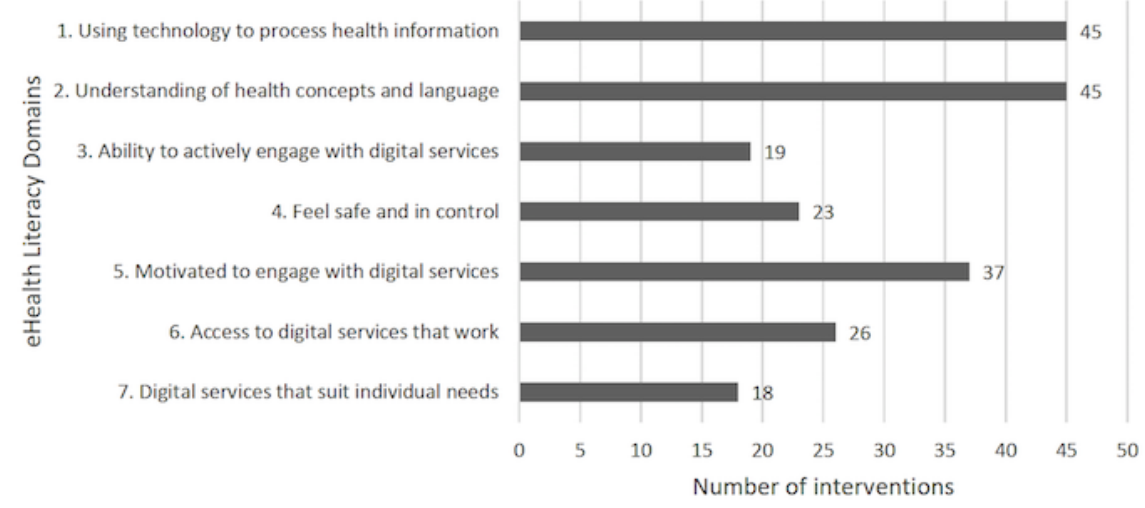

\section{Effectiveness of eHealth Interventions}

Although no study explicitly considered or assessed eHealth literacy, the effectiveness of eHealth interventions was nevertheless examined, and the results were mixed. Approximately one-fourth of the reviewed interventions $(n=13)$ did not find statistically significant improvements in their primary outcomes. Although 19 studies reported significant improvements in their primary outcomes, another 12 studies found significant improvements in some primary outcomes, but not all. In addition, 4 studies found improvements but did not report whether such differences were significant. The results of long-term effectiveness also produced mixed evidence. Among the 13 studies that conducted follow-up assessments, 8 found the effects sustainable up to a period of 12 months. A total of 3 studies found effects were not sustainable, whereas 2 did not report on the significance (Multimedia Appendix 3).

Among the reported effective 19 studies, there were no consistent patterns of intervention characteristics or eHealth literacy domains likely addressed. These interventions could be interactive or noninteractive, although platforms and devices also varied. The number of eHealth literacy domains likely addressed ranged from 1 to 7 . Although a study by King et al [65] likely addressed all 7 domains and found their intervention effective with a large effect size (0.8-1.2), a study by Chen et al [48] also reported their study as effective, although only the domain 1. Using technology to process health information could be identified within the intervention components.

\section{Discussion}

\section{Principal Findings}

Although the concept of eHealth literacy was introduced more than a decade ago [17], this review finds that utilization of the concept for enhancing eHealth use and engagement is rarely recognized. The eHealth literacy needs of users were not explicitly considered during intervention development in any of the included studies, and no eHealth literacy assessment was conducted to ensure that such needs were met. This result is echoed in an earlier systematic review of eHealth and telehealth tools for vulnerable populations, which reported that eHealth literacy was not assessed in any of the 18 included studies [95]. In fact, eHealth literacy is only mentioned in 1 of the 51 papers of this review. Although 3 studies conducted health literacy assessments at baseline [71,73,75] and 1 assessed eHealth literacy as a secondary outcome [45], the results were not used for intervention development. The fact that eHealth literacy is overlooked may be because of the lack of comprehensive measures before 2018 and an associated knowledge gap in using such assessment to inform eHealth design [96]. To move forward, in-depth research on eHealth literacy is required, such as the application of the eHLF and the recently developed comprehensive eHLQ, designed to support eHealth intervention development and evaluation. Developed on the basis of eHLF, the eHLQ is a 35 -item questionnaire that produces 7 scores representing 7 eHealth literacy domains of users. The resulting scores provide insights into users' strengths and weaknesses in using eHealth such that interventions can be tailored accordingly. For example, if target users reported good ability to use technology (higher scores in 3. Ability to actively engage with digital services) but lack motivation (lower scores in 5 . Motivated to engage with digital services), features to address motivation should be a prominent feature of the intervention. However, if target users demonstrated limited ability to use technologies, interventions such as simple unidirectional text messages, rather than interactive mobile apps, are likely to be more suitable for the target users. Hence, the eHLF and eHLQ will have the potential to advance the field of eHealth literacy and strengthen the reach and impact of digital health interventions [25].

\section{Addressing eHealth Literacy Needs}

There is growing concern that frameworks or guidelines informing the development of eHealth interventions so that they meet users' needs are lacking [97], and this concern is reflected in the findings of this review. Only 1 study [77] used the Research-based Web Design and Usability Guidelines by the UDHHS to inform intervention development. However, the guideline authors specifically indicate that they may not be applicable to all audiences, such as people with low literacy who may have different reading and layout needs [94]. In addition, only 22 studies in this review discussed user involvement whereas needs assessments were usually in the form of focus groups or interviews involving a limited number of users. Only 1 study reported the inclusion of patients in content writing [37]. Such practice means that interventions are expert driven instead of user driven, echoing the concern that users and patients are the most underused resources in developing eHealth interventions [98]. Although eHealth literacy 
is only one of the factors in developing effective eHealth strategies, it has been advocated that it is a primary and critical factor that affects usability and adoption $[17,21,26]$. Even if an intervention is grounded in theory, it will not be usable if it does not align with the literacy needs and abilities of end users and may lead to nonadoption $[30,99,100]$. Hence, research efforts into eHealth developmental frameworks incorporating eHealth literacy need assessment, and user-centered principles are required such that equal access and usage can be achieved for all users.

Although eHealth literacy needs may not be explicitly considered when developing eHealth programs, this review still finds that interventions generally have features that may meet eHealth literacy needs based on the eHLF. However, the common focus is on providing information or features that address the domains of 1 . Using technology to process health information, 2. Understanding of health concepts and language, and 5. Motivated to engage with digital services. Strategies to assist users in using or engaging with technology and accessibility features of systems that are tailored and responsive to an individual's ability and capability are generally overlooked. These findings resonate with those from a systematic review of diabetes apps targeted at older adults that there is a limited variety of accessibility features [101]. This is of special importance when an intervention is designed for older people or people with disabilities who may require specialized tools because of functional and cognitive impairments [102,103] or people with low literacy skills who may have different reading and design needs [94]. In addition, applying the eHLF to determine whether certain eHealth literacy domains were addressed may not necessarily mean that the eHealth literacy needs of users were met as the actual eHealth literacy needs of target users were not assessed and, therefore, not known. The results highlight that in developing interventions using technologies, designers are mainly responsible for ensuring that users' needs and capabilities are met in the hope that users will adopt the intervention to improve or change their health behavior. However, Chang et al [104] noted that eHealth intervention designers were typically not trained to meet the communication needs of underserved communities. Showell et al [105] also pointed out that eHealth systems tended to be designed for users who were similar to the designers, who were usually middle-class professionals. As such, the needs of disadvantaged patients were generally overlooked in the design process [15].

\section{Effectiveness of eHealth Interventions}

In addition to exploring the role of eHealth literacy and eHealth intervention development, this review also examined the effectiveness of eHealth interventions targeted at socially disadvantaged groups and found inconclusive evidence. Although significant improvements were found in 19 studies, these findings should be interpreted with caution, as 10 studies are of weak quality and 7 studies are of moderate quality. Although 3 studies reported a large effect size, they had smaller sample sizes and were of moderate or weak quality [51,65,83]. The sustainability of effects is also mixed and cannot be ascertained, as most studies have short follow-up times. These findings are similar to reviews of eHealth interventions, which also report inconclusive evidence on effectiveness $[35,103,106,107]$. The lack of comprehensive eHealth literacy assessments also prevents this review from exploring the link between eHealth literacy and the effectiveness of eHealth programs. Further robust empirical studies need to be undertaken to better understand the role of eHealth literacy in eHealth interventions to help address the digital divide and improve health disparities.

\section{Limitations}

Several limitations of this review need to be acknowledged. Only peer-reviewed journals were included for this review, and there may be other studies that were not accounted for. The search was conducted by one researcher, which may have led to potential bias. The findings of this review may not reflect all details of the actual intervention, as authors generally only briefly describe their intervention development processes [108], and few studies report how users are involved such that interventions are aligned with their needs [27]. However, not reporting certain features suggests that the authors may not consider such features as relevant. Furthermore, the included studies do not represent all socially disadvantaged groups. This review only focused on certain categories of disadvantaged groups and did not include other underserved populations, such as people with disabilities or indigenous people who may also have limited access or skills to use ICT [109]. Future reviews should consider inclusion of these groups to advance eHealth research among vulnerable populations.

\section{Conclusions}

The WHO recognizes health literacy as a critical determinant of health that has the potential to empower individuals and bring about health equity [19]. However, this systematic review finds that the role of eHealth literacy in designing eHealth interventions targeted at socially disadvantaged groups is generally overlooked. eHealth literacy was not explicitly considered or assessed during intervention development. There was also a lack of frameworks or theories informing eHealth designers on how to meet users' needs. Although users were involved in some of the reviewed studies, intervention development was mainly expert driven rather than user driven. By using the eHLF to examine the eHealth literacy components of eHealth interventions, it was found that the design of features such that they suited individual capability was not common. Furthermore, whether the eHealth literacy needs of users were actually addressed in the reviewed interventions cannot be ascertained because of the lack of comprehensive eHealth literacy assessment. The link between eHealth literacy and effectiveness of eHealth interventions cannot be explored. Moreover, the paucity of robust studies also delivers limited empirical evidence on how to effectively reach these vulnerable populations and bridge the digital divide.

Despite the concept of eHealth literacy being introduced in 2006, its potential role in empowering individuals has not been realized. Without meeting the eHealth literacy needs of disadvantaged groups, adoption of eHealth interventions is likely to be low, resulting in ineffective interventions $[17,21,30,99,100,110]$. To ensure that no one is left behind as determined in the Shanghai Declaration on Promoting Health 
[19], eHealth literacy must be acknowledged and included in the development of eHealth interventions to assist the realization

of technological advancement and improve health equity.

\section{Acknowledgments}

The authors would like to thank Associate Professor Lars Kayser, University of Copenhagen; Assistant Professor Sarah Fredsted Villadsen, University of Copenhagen; and Dr Nancy Huang, Chief Medical Advisor, Australian Unity for their contribution during the conceptualization stage of this systematic review. The authors would also like to thank Dr Polina Putrik, Visiting Fellow at Deakin University, for her input in search strategy. RO was funded in part through the National Health and Medical Research Council of Australia Principal Research Fellowship \#APP1155125.

\section{Conflicts of Interest}

None declared.

\section{Multimedia Appendix 1}

Search strategies.

[DOCX File, 20 KB-Multimedia Appendix 1]

\section{Multimedia Appendix 2}

Quality assessment.

[DOCX File, 27 KB-Multimedia Appendix 2]

\section{Multimedia Appendix 3}

Intervention characteristics and key findings. [DOCX File, $50 \mathrm{~KB}$-Multimedia Appendix 3]

\section{Multimedia Appendix 4}

Summary of eHealth literacy domains likely addressed.

[DOCX File, 54 KB-Multimedia Appendix 4]

\section{References}

1. World Health Organization. WHO Guideline: Recommendations on Digital Interventions for Health System Strengthening. Geneva, Switzerland: World Health Organization; 2019.

2. Digital Health. World Health Organization. 2018. URL: http://apps.who.int/gb/ebwha/pdf files/WHA71/A71 R7-en. pdf?ua=1 [accessed 2020-03-19]

3. Bodie GD, Dutta MJ. Understanding health literacy for strategic health marketing: eHealth literacy, health disparities, and the digital divide. Health Mark Q 2008;25(1-2):175-203. [doi: 10.1080/07359680802126301] [Medline: 18935884]

4. Litt E. Measuring users' internet skills: a review of past assessments and a look toward the future. New Media Soc 2013 May 24;15(4):612-630. [doi: 10.1177/1461444813475424] [Medline: $\underline{87775130]}$

5. Falling Through the Net: Defining the Digital Divide, A Report on the Telecommunications and Information Technology Gap in America. Washington, DC: US Department of Commerce; 1999.

6. Wei K, Teo H, Chan H, Tan B. Conceptualizing and testing a social cognitive model of the digital divide. Inf Syst Res 2011 Mar 22;22(1):170-187 [FREE Full text] [doi: 10.1287/isre.1090.0273]

7. Ginossar T. Predictors of online cancer prevention information seeking among patients and caregivers across the digital divide: a cross-sectional, correlational study. JMIR Cancer 2016 Mar 9;2(1):e2 [FREE Full text] [doi: 10.2196/cancer.5108] [Medline: 28410177]

8. Lustria ML, Smith SA, Hinnant CC. Exploring digital divides: an examination of eHealth technology use in health information seeking, communication and personal health information management in the USA. Health Informatics J 2011 Sep;17(3):224-243. [doi: 10.1177/1460458211414843] [Medline: 21937464]

9. Asoh DA, Rivers PA. The empowerment and quality health value propositions of e-health. Health Serv Manage Res 2010 Nov;23(4):181-184. [doi: 10.1258/hsmr.2010.010007] [Medline: 21097729]

10. Viswanath K, Kreuter MW. Health disparities, communication inequalities, and eHealth. Am J Prev Med 2007 May;32(5 Suppl):S131-S133 [FREE Full text] [doi: 10.1016/j.amepre.2007.02.012] [Medline: 17466818]

11. Baur C. An analysis of factors underlying e-health disparities. Camb Q Healthc Ethics 2008;17(4):417-428. [doi: 10.1017/S0963180108080547] [Medline: 18724881] 
12. Kontos E, Blake KD, Chou WY, Prestin A. Predictors of eHealth usage: insights on the digital divide from the health information national trends survey 2012. J Med Internet Res 2014 Jul 16;16(7):e172 [FREE Full text] [doi: 10.2196/jmir.3117] [Medline: 25048379]

13. Choi NG, Dinitto DM. The digital divide among low-income homebound older adults: internet use patterns, eHealth literacy, and attitudes toward computer/internet use. J Med Internet Res 2013 May 2;15(5):e93 [FREE Full text] [doi: 10.2196/jmir.2645] [Medline: 23639979]

14. Kim E, Stolyar A, Lober WB, Herbaugh AL, Shinstrom SE, Zierler BK, et al. Challenges to using an electronic personal health record by a low-income elderly population. J Med Internet Res 2009 Oct 27;11(4):e44 [REEE Full text] [doi: 10.2196/jmir.1256] [Medline: 19861298]

15. Showell C, Cummings E, Turner P. The invisibility of disadvantage: why do we not notice? Stud Health Technol Inform 2017;235:388-392. [Medline: 28423820]

16. Hasnain-Wynia R, Wolf MS. Promoting health care equity: is health literacy a missing link? Health Serv Res 2010 Aug;45(4):897-903 [FREE Full text] [doi: 10.1111/j.1475-6773.2010.01134.x] [Medline: 20646073]

17. Norman CD, Skinner HA. Ehealth literacy: essential skills for consumer health in a networked world. J Med Internet Res 2006 Jun 16;8(2):e9 [FREE Full text] [doi: 10.2196/jmir.8.2.e9] [Medline: 16867972]

18. Austin R. EHealth Literacy for Older Adults - Part II. EBSCOhost Connection. 2012. URL: http://connection.ebscohost.com/ c/articles/79632384/ehealth-literacy-older-adults-part-ii [accessed 2020-07-28]

19. World Health Organization. Shanghai declaration on promoting health in the 2030 agenda for sustainable development. Health Promot Int 2017 Feb 1;32(1):7-8. [doi: 10.1093/heapro/daw103] [Medline: 28180270]

20. Norman C. eHealth literacy 2.0: problems and opportunities with an evolving concept. J Med Internet Res 2011 Dec 23;13(4):e125 [FREE Full text] [doi: 10.2196/jmir.2035] [Medline: 22193243]

21. Kayser L, Kushniruk A, Osborne RH, Norgaard O, Turner P. Enhancing the effectiveness of consumer-focused health information technology systems through ehealth literacy: a framework for understanding users' needs. JMIR Hum Factors 2015 May 20;2(1):e9 [FREE Full text] [doi: 10.2196/humanfactors.3696] [Medline: 27025228]

22. Monkman H, Griffith J, Kushniruk A. Evidence-based heuristics for evaluating demands on ehealth literacy and usability in a mobile consumer health application. Stud Health Technol Inform 2015;216:358-362. [Medline: 26262071]

23. Norgaard O, Furstand D, Klokker L, Karnoe A, Batterham R, Kayser L. The e-health literacy framework: a conceptual framework for characterizing e-health users and their interaction with e-health systems. Know Manag E-Learn 2015;7(4):522-540. [doi: 10.34105/j.kmel.2015.07.035]

24. Buchbinder R, Batterham B, Elsworth G, Dionne CE, Irvin E, Osborne RH. A validity-driven approach to the understanding of the personal and societal burden of low back pain: development of a conceptual and measurement model. Arthritis Res Ther 2011;13(5):R152 [FREE Full text] [doi: 10.1186/ar3468] [Medline: 21933393]

25. Kayser L, Karnoe A, Furstrand D, Batterham R, Christensen KB, Elsworth G, et al. A multidimensional tool based on the ehealth literacy framework: development and initial validity testing of the ehealth literacy questionnaire (EHLQ). J Med Internet Res 2018 Feb 12;20(2):e36 [FREE Full text] [doi: 10.2196/jmir.8371] [Medline: 29434011]

26. Monkman H, Kushniruk A. eHealth literacy issues, constructs, models, and methods for health information technology design and evaluation. Know Manag E-Learn 2015;7(4):541-549. [doi: 10.34105/j.kmel.2015.07.036]

27. de vito Dabbs A, Myers BA, Mc Curry KR, Dunbar-Jacob J, Hawkins RP, Begey A, et al. User-centered design and interactive health technologies for patients. Comput Inform Nurs 2009;27(3):175-183 [FREE Full text] [doi: 10.1097/NCN.0b013e31819f7c7c] [Medline: 19411947]

28. LeRouge C, Ma J, Sneha S, Tolle K. User profiles and personas in the design and development of consumer health technologies. Int J Med Inform 2013 Nov;82(11):e251-e268. [doi: 10.1016/j.ijmedinf.2011.03.006] [Medline: 21481635]

29. Roehrer E, Cummings E, Ellis L, Turner P. The role of user-centred design within online community development. Stud Health Technol Inform 2011;164:256-260. [Medline: 21335720]

30. Greenhalgh T, Hinder S, Stramer K, Bratan T, Russell J. Adoption, non-adoption, and abandonment of a personal electronic health record: case study of HealthSpace. Br Med J 2010 Nov 16;341:c5814 [FREE Full text] [doi: 10.1136/bmj.c5814] [Medline: 21081595]

31. Moher D, Shamseer L, Clarke M, Ghersi D, Liberati A, Petticrew M, PRISMA-P Group. Preferred reporting items for systematic review and meta-analysis protocols (PRISMA-P) 2015 statement. Syst Rev 2015 Jan 1;4:1 [FREE Full text] [doi: 10.1186/2046-4053-4-1] [Medline: 25554246]

32. Schardt C, Adams MB, Owens T, Keitz S, Fontelo P. Utilization of the PICO framework to improve searching PubMed for clinical questions. BMC Med Inform Decis Mak 2007 Jun 15;7:16 [FREE Full text] [doi: 10.1186/1472-6947-7-16] [Medline: 17573961]

33. Physical Activity Promotion In Socially Disadvantaged Groups: Principles for Action. WHO/Europe. 2013. URL: http:/ /www.euro.who.int/_data/assets/pdf_file/0006/193092/PHAN-brochure_ENG.pdf [accessed 2020-11-03]

34. World Population Prospects: The 2017 Revision: Key Findings and Advance Tables. United Nations Population Division. 2017. URL: https://esa.un.org/unpd/wpp/Publications/Files/WPP2017 KeyFindings.pdf [accessed 2020-11-03] 
35. Bennett GG, Steinberg DM, Stoute C, Lanpher M, Lane I, Askew S, et al. Electronic health (eHealth) interventions for weight management among racial/ethnic minority adults: a systematic review. Obes Rev 2014 Oct;15(Suppl 4):146-158. [doi: 10.1111/obr.12218] [Medline: 25196411]

36. Quality Assessment Tool for Quantitative Studies. Canadian Health Care. 2009. URL: https://www.ephpp.ca/ quality-assessment-tool-for-quantitative-studies/ [accessed 2017-12-03]

37. Agyapong VI, Juhás M, Ohinmaa A, Omeje J, Mrklas K, Suen VY, et al. Randomized controlled pilot trial of supportive text messages for patients with depression. BMC Psychiatry 2017 Aug 2;17(1):286 [FREE Full text] [doi: 10.1186/s12888-017-1448-2] [Medline: 28768493]

38. Anand SS, Samaan Z, Middleton C, Irvine J, Desai D, Schulze KM, South Asian Heart Risk Assessment Investigators. A digital health intervention to lower cardiovascular risk: a randomized clinical trial. JAMA Cardiol 2016 Aug 1;1(5):601-606. [doi: 10.1001/jamacardio.2016.1035] [Medline: 27438754]

39. Arora S, Peters AL, Burner E, Lam CN, Menchine M. Trial to examine text message-based mHealth in emergency department patients with diabetes (TExT-MED): a randomized controlled trial. Ann Emerg Med 2014 Jun;63(6):745-54.e6. [doi: 10.1016/j.annemergmed.2013.10.012] [Medline: 24225332]

40. Bennett GG, Steinberg D, Askew S, Levine E, Foley P, Batch BC, et al. Effectiveness of an app and provider counseling for obesity treatment in primary care. Am J Prev Med 2018 Dec;55(6):777-786 [FREE Full text] [doi:

10.1016/j.amepre.2018.07.005] [Medline: $\underline{\text { 30361140] }}$

41. Bond GE, Burr RL, Wolf FM, Feldt K. The effects of a web-based intervention on psychosocial well-being among adults aged 60 and older with diabetes: a randomized trial. Diabetes Educ 2010;36(3):446-456. [doi: 10.1177/0145721710366758] [Medline: 20375351]

42. Broekhuizen K, de Gelder J, Wijsman CA, Wijsman LW, Westendorp RG, Verhagen E, et al. An internet-based physical activity intervention to improve quality of life of inactive older adults: a randomized controlled trial. J Med Internet Res 2016 Apr 27;18(4):e74 [FREE Full text] [doi: 10.2196/jmir.4335] [Medline: 27122359]

43. Wijsman CA, Westendorp RG, Verhagen EA, Catt M, Slagboom PE, de Craen AJ, et al. Effects of a web-based intervention on physical activity and metabolism in older adults: randomized controlled trial. J Med Internet Res 2013 Nov 6;15(11):e233 [FREE Full text] [doi: 10.2196/jmir.2843] [Medline: 24195965]

44. Buller DB, Woodall WG, Zimmerman DE, Slater MD, Heimendinger J, Waters E, et al. Randomized trial on the 5 a day, the Rio Grande Way Website, a web-based program to improve fruit and vegetable consumption in rural communities. $\mathbf{J}$ Health Commun 2008;13(3):230-249 [FREE Full text] [doi: 10.1080/10810730801985285] [Medline: 18569356]

45. Carroll JK, Tobin JN, Luque A, Farah S, Sanders M, Cassells A, et al. 'Get ready and empowered about rreatment' (GREAT) study: a pragmatic randomized controlled trial of activation in persons living with HIV. J Gen Intern Med 2019 Sep;34(9):1782-1789. [doi: 10.1007/s11606-019-05102-7] [Medline: 31240605]

46. Caster MM, Norris AH, Butao C, Carr Reese P, Chemey E, Phuka J, et al. Assessing the acceptability, feasibility, and effectiveness of a tablet-based cervical cancer educational intervention. J Cancer Educ 2017 Mar;32(1):35-42 [FREE Full text] [doi: 10.1007/s13187-015-0953-6] [Medline: 26637473]

47. Chen YX, Hung YP, Chen HC. Mobile application-assisted cognitive behavioral therapy for insomnia in an older adult. Telemed J E Health 2016 Apr;22(4):332-334. [doi: 10.1089/tmj.2015.0064] [Medline: 26393902]

48. Chen T, Zhu W, Tang B, Jin L, Fu H, Chen Y, et al. A mobile phone informational reminder to improve eye care adherence among diabetic patients in rural China: a randomized controlled trial. Am J Ophthalmol 2018 Oct;194:54-62. [doi: 10.1016/j.ajo.2018.07.006] [Medline: 30053472]

49. Choi I, Zou J, Titov N, Dear BF, Li S, Johnston L, et al. Culturally attuned Internet treatment for depression amongst Chinese Australians: a randomised controlled trial. J Affect Disord 2012 Feb;136(3):459-468. [doi: 10.1016/j.jad.2011.11.003] [Medline: 22177742]

50. Dang S, Karanam C, Gómez-Marín O. Outcomes of a mobile phone intervention for heart failure in a minority county hospital population. Telemed J E Health 2017 Jun;23(6):473-484. [doi: 10.1089/tmj.2016.0211] [Medline: 28051357]

51. Dear BF, Zou JB, Ali S, Lorian CN, Johnston L, Sheehan J, et al. Clinical and cost-effectiveness of therapist-guided internet-delivered cognitive behavior therapy for older adults with symptoms of anxiety: a randomized controlled trial. Behav Ther 2015 Mar;46(2):206-217. [doi: 10.1016/j.beth.2014.09.007] [Medline: 25645169]

52. Dugas M, Crowley K, Gao GG, Xu T, Agarwal R, Kruglanski AW, et al. Individual differences in regulatory mode moderate the effectiveness of a pilot mHealth trial for diabetes management among older veterans. PLoS One 2018;13(3):e0192807 [FREE Full text] [doi: 10.1371/journal.pone.0192807] [Medline: 29513683]

53. Fortmann AL, Gallo LC, Garcia MI, Taleb M, Euyoque JA, Clark T, et al. Dulce digital: an mhealth SMS-based intervention improves glycemic control in Hispanics with type 2 diabetes. Diabetes Care 2017 Oct;40(10):1349-1355 [FREE Full text] [doi: 10.2337/dc17-0230] [Medline: 28600309]

54. Gilmore LA, Klempel MC, Martin CK, Myers CA, Burton JH, Sutton EF, et al. Personalized mobile health intervention for health and weight loss in postpartum women receiving women, infants, and children benefit: a randomized controlled pilot study. J Womens Health (Larchmt) 2017 Jul;26(7):719-727 [FREE Full text] [doi: 10.1089/jwh.2016.5947] [Medline: 28338403] 
55. Griffin JB, Struempler B, Funderburk K, Parmer SM, Tran C, Wadsworth DD. My quest, an intervention using text messaging to improve dietary and physical activity behaviors and promote weight loss in low-income women. J Nutr Educ Behav 2018 Jan;50(1):11-8.e1. [doi: 10.1016/j.jneb.2017.09.007] [Medline: 29325657]

56. Hacking D, Haricharan HJ, Brittain K, Lau YK, Cassidy T, Heap M. Hypertension health promotion via text messaging at a community health center in South Africa: a mixed methods study. JMIR Mhealth Uhealth 2016 Mar 10;4(1):e22 [FREE Full text] [doi: 10.2196/mhealth.4569] [Medline: 26964505]

57. Hageman PA, Pullen CH, Hertzog M, Boeckner LS. Effectiveness of tailored lifestyle interventions, using web-based and print-mail, for reducing blood pressure among rural women with prehypertension: main results of the wellness for women: dashing towards health clinical trial. Int J Behav Nutr Phys Act 2014 Dec 6;11:148 [FREE Full text] [doi: 10.1186/s12966-014-0148-2] [Medline: 25480461]

58. Herring SJ, Cruice JF, Bennett GG, Darden N, Wallen JJ, Rose MZ, et al. Intervening during and after pregnancy to prevent weight retention among African American women. Prev Med Rep 2017 Sep;7:119-123 [FREE Full text] [doi: 10.1016/j.pmedr.2017.05.015] [Medline: 28660118]

59. Hill W, Weinert C, Cudney S. Influence of a computer intervention on the psychological status of chronically ill rural women: preliminary results. Nurs Res 2006;55(1):34-42 [FREE Full text] [doi: 10.1097/00006199-200601000-00005] [Medline: 16439927]

60. Hong YA, Goldberg D, Ory MG, Towne SD, Forjuoh SN, Kellstedt D, et al. Efficacy of a mobile-enabled web app (iCanFit) in promoting physical activity among older cancer survivors: a pilot study. JMIR Cancer 2015 Jun 26;1(1):e7 [FREE Full text] [doi: 10.2196/cancer.4389] [Medline: 28410158]

61. Ingersoll KS, Dillingham RA, Hettema JE, Conaway M, Freeman J, Reynolds G, et al. Pilot RCT of bidirectional text messaging for ART adherence among nonurban substance users with HIV. Health Psychol 2015 Dec;34S:1305-1315 [FREE Full text] [doi: 10.1037/hea0000295] [Medline: 26651472]

62. Jarvis MA, Padmanabhanunni A, Chipps J. An evaluation of a low-intensity cognitive behavioral therapy mhealth-supported intervention to reduce loneliness in older people. Int J Environ Res Public Health 2019 Apr 11;16(7):1-14 [FREE Full text] [doi: 10.3390/ijerph16071305] [Medline: 30979042]

63. Joseph RP, Keller C, Adams MA, Ainsworth BE. Print versus a culturally-relevant Facebook and text message delivered intervention to promote physical activity in African American women: a randomized pilot trial. BMC Womens Health 2015 Mar 27;15:30 [FREE Full text] [doi: 10.1186/s12905-015-0186-1] [Medline: 25886945]

64. Kamal AK, Shaikh Q, Pasha O, Azam I, Islam M, Memon AA, et al. A randomized controlled behavioral intervention trial to improve medication adherence in adult stroke patients with prescription tailored short messaging service (SMS)-SMS4Stroke study. BMC Neurol 2015 Oct 21;15:212 [FREE Full text] [doi: 10.1186/s12883-015-0471-5] [Medline: 26486857]

65. King AC, Bickmore TW, Campero MI, Pruitt LA, Yin JL. Employing virtual advisors in preventive care for underserved communities: results from the COMPASS study. J Health Commun 2013;18(12):1449-1464 [FREE Full text] [doi: 10.1080/10810730.2013.798374] [Medline: 23941610]

66. Kiropoulos LA, Griffiths KM, Blashki G. Effects of a multilingual information website intervention on the levels of depression literacy and depression-related stigma in Greek-born and Italian-born immigrants living in Australia: a randomized controlled trial. J Med Internet Res 2011 Apr 19;13(2):e34 [FREE Full text] [doi: 10.2196/jmir.1527] [Medline: 21504872]

67. Lee HY, Koopmeiners JS, Rhee TG, Raveis VH, Ahluwalia JS. Mobile phone text messaging intervention for cervical cancer screening: changes in knowledge and behavior pre-post intervention. J Med Internet Res 2014 Aug 27;16(8):e196 [FREE Full text] [doi: 10.2196/jmir.3576] [Medline: 25164545]

68. Lee HY, Koopmeiners JS, McHugh J, Raveis VH, Ahluwalia JS. Mhealth pilot study: text messaging intervention to promote HPV vaccination. Am J Health Behav 2016 Jan;40(1):67-76 [FREE Full text] [doi: 10.5993/AJHB.40.1.8] [Medline: 26685815]

69. Lee H, Ghebre R, Le C, Jang YJ, Sharratt M, Yee D. Mobile phone multilevel and multimedia messaging intervention for breast cancer screening: pilot randomized controlled trial. JMIR Mhealth Uhealth 2017 Nov 7;5(11):e154 [FREE Full text] [doi: 10.2196/mhealth.7091] [Medline: 29113961]

70. MacDonell KK, Naar S, Gibson-Scipio W, Lam P, Secord E. The Detroit young adult asthma project: pilot of a technology-based medication adherence intervention for African-American emerging adults. J Adolesc Health 2016 Oct;59(4):465-471 [FREE Full text] [doi: 10.1016/j.jadohealth.2016.05.016] [Medline: 27475032]

71. Marcus BH, Hartman SJ, Larsen BA, Pekmezi D, Dunsiger SI, Linke S, et al. Pasos Hacia La Salud: a randomized controlled trial of an internet-delivered physical activity intervention for Latinas. Int J Behav Nutr Phys Act 2016 May 28;13:62 [FREE Full text] [doi: 10.1186/s12966-016-0385-7] [Medline: 27234302]

72. Mauriello LM, van Marter DF, Umanzor CD, Castle PH, de Aguiar EL. Using mhealth to deliver behavior change interventions within prenatal care at community health centers. Am J Health Promot 2016 Sep;30(7):554-562. [doi: 10.4278/ajhp.140530-QUAN-248] [Medline: 26305603]

73. Miller DP, Denizard-Thompson N, Weaver KE, Case LD, Troyer JL, Spangler JG, et al. Effect of a digital health intervention on receipt of colorectal cancer screening in vulnerable patients: a randomized controlled trial. Ann Intern Med 2018 Apr 17;168(8):550-557 [FREE Full text] [doi: 10.7326/M17-2315] [Medline: 29532054] 
74. Moussa M, Sherrod D, Choi J. An e-health intervention for increasing diabetes knowledge in African Americans. Int J Nurs Pract 2013 Sep;19(Suppl 3):36-43. [doi: 10.1111/ijn.12167] [Medline: 24090296]

75. Neafsey PJ, M'lan CE, Ge M, Walsh SJ, Lin CA, Anderson E. Reducing adverse self-medication behaviors in older adults with hypertension: results of an e-health clinical efficacy trial. Ageing Int 2011 Jun;36(2):159-191 [FREE Full text] [doi: 10.1007/s12126-010-9085-9] [Medline: 21654869]

76. Nelson LA, Mulvaney SA, Gebretsadik T, Johnson KB, Osborn CY. The messaging for diabetes (MED) intervention improves short-term medication adherence among low-income adults with type 2 diabetes. J Behav Med 2016 Dec;39(6):995-1000 [FREE Full text] [doi: 10.1007/s10865-016-9774-2] [Medline: 27488604]

77. Neuenschwander LM, Abbott A, Mobley AR. Comparison of a web-based vs in-person nutrition education program for low-income adults. J Acad Nutr Diet 2013 Jan;113(1):120-126. [doi: 10.1016/j.jand.2012.07.034] [Medline: 23092741]

78. Phelan S, Hagobian T, Brannen A, Hatley KE, Schaffner A, Muñoz-Christian K, et al. Effect of an internet-based program on weight loss for low-income postpartum women: a randomized clinical trial. J Am Med Assoc 2017 Jun 20;317(23):2381-2391 [FREE Full text] [doi: 10.1001/jama.2017.7119] [Medline: 28632867]

79. Rubinstein A, Miranda JJ, Beratarrechea A, Diez-Canseco F, Kanter R, Gutierrez L, GISMAL group. Effectiveness of an mHealth intervention to improve the cardiometabolic profile of people with prehypertension in low-resource urban settings in Latin America: a randomised controlled trial. Lancet Diabetes Endocrinol 2016 Jan;4(1):52-63. [doi: 10.1016/S2213-8587(15)00381-2] [Medline: 26653067]

80. Ryan JG, Schwartz R, Jennings T, Fedders M, Vittoria I. Feasibility of an internet-based intervention for improving diabetes outcomes among low-income patients with a high risk for poor diabetes outcomes followed in a community clinic. Diabetes Educ 2013;39(3):365-375. [doi: 10.1177/0145721713484594] [Medline: 23610181]

81. Steinberg DM, Levine EL, Askew S, Foley P, Bennett GG. Daily text messaging for weight control among racial and ethnic minority women: randomized controlled pilot study. J Med Internet Res 2013 Nov 18;15(11):e244 [FREE Full text] [doi: 10.2196/jmir.2844] [Medline: 24246427]

82. Tessaro I, Rye S, Parker L, Mangone C, McCrone S. Effectiveness of a nutrition intervention with rural low-income women. Am J Health Behav 2007;31(1):35-43. [doi: 10.5555/ajhb.2007.31.1.35] [Medline: 17181460]

83. Titov N, Dear BF, Ali S, Zou JB, Lorian CN, Johnston L, et al. Clinical and cost-effectiveness of therapist-guided internet-delivered cognitive behavior therapy for older adults with symptoms of depression: a randomized controlled trial. Behav Ther 2015 Mar;46(2):193-205. [doi: 10.1016/j.beth.2014.09.008] [Medline: 25645168]

84. Ünlü Ince B, Cuijpers P, van 't Hof E, van Ballegooijen W, Christensen H, Riper H. Internet-based, culturally sensitive, problem-solving therapy for Turkish migrants with depression: randomized controlled trial. J Med Internet Res 2013 Oct 11;15(10):e227 [FREE Full text] [doi: 10.2196/jmir.2853] [Medline: 24121307]

85. Wahbeh H, Goodrich E, Oken B. Internet-based mindfulness meditation for cognition and mood in older adults: a pilot study. Altern Ther Health Med 2016;22(2):44-53 [FREE Full text] [Medline: 27036056]

86. Wayne N, Perez DF, Kaplan DM, Ritvo P. Health coaching reduces HbA1c in type 2 diabetic patients from a lower-socioeconomic status community: a randomized controlled trial. J Med Internet Res 2015 Oct 5;17(10):e224 [FREE Full text] [doi: 10.2196/jmir.4871] [Medline: 26441467]

87. Weinert C, Cudney S, Hill W. Health knowledge acquisition by rural women with chronic health conditions: a tale of two web approaches. Aust J Rural Health 2008 Oct;16(5):302-307 [FREE Full text] [doi: 10.1111/j.1440-1584.2008.01004.x] [Medline: 18808489$]$

88. Armijo-Olivo S, Stiles CR, Hagen NA, Biondo PD, Cummings GG. Assessment of study quality for systematic reviews: a comparison of the cochrane collaboration risk of bias tool and the effective public health practice project quality assessment tool: methodological research. J Eval Clin Pract 2012 Feb;18(1):12-18. [doi: 10.1111/j.1365-2753.2010.01516.x] [Medline: 20698919]

89. Thomas BH, Ciliska D, Dobbins M, Micucci S. A process for systematically reviewing the literature: providing the research evidence for public health nursing interventions. Worldviews Evid Based Nurs 2004;1(3):176-184. [doi:

10.1111/j.1524-475X.2004.04006.x] [Medline: 17163895]

90. Hsieh H, Shannon SE. Three approaches to qualitative content analysis. Qual Health Res 2005 Nov;15(9):1277-1288. [doi: 10.1177/1049732305276687] [Medline: 16204405]

91. Karnoe A, Kayser L. How is eHealth literacy measured and what do the measurements tell us? A systematic review. Know Manag E-Learn 2015;7(4):576-600. [doi: 10.34105/j.kmel.2015.07.038]

92. Taylor VM, Jackson JC, Tu S, Yasui Y, Schwartz SM, Kuniyuki A, et al. Cervical cancer screening among Chinese Americans. Cancer Detect Prev 2002;26(2):139-145 [FREE Full text] [doi: 10.1016/s0361-090x(02)00037-5] [Medline: $\underline{12102148]}$

93. Norman CD, Skinner HA. eHEALS: the eHealth literacy scale. J Med Internet Res 2006 Nov 14;8(4):e27 [FREE Full text] [doi: 10.2196/jmir.8.4.e27] [Medline: 17213046]

94. Research-based Web Design and Usability Guidelines. The US Department of Health and Human Services (HHS). 2004. URL: https://webstandards.hhs.gov/guidelines/ [accessed 2019-03-18] 
95. Parker S, Prince A, Thomas L, Song H, Milosevic D, Harris MF, IMPACT Study Group. Electronic, mobile and telehealth tools for vulnerable patients with chronic disease: a systematic review and realist synthesis. BMJ Open 2018 Aug 29;8(8):e019192 [FREE Full text] [doi: 10.1136/bmjopen-2017-019192] [Medline: 30158214]

96. Griebel L, Enwald H, Gilstad H, Pohl AL, Moreland J, Sedlmayr M. eHealth literacy research-Quo vadis? Inform Health Soc Care 2018 Dec;43(4):427-442. [doi: 10.1080/17538157.2017.1364247] [Medline: 29045164]

97. Mohr DC, Schueller SM, Montague E, Burns MN, Rashidi P. The behavioral intervention technology model: an integrated conceptual and technological framework for eHealth and mHealth interventions. J Med Internet Res 2014 Jun 5;16(6):e146 [FREE Full text] [doi: 10.2196/jmir.3077] [Medline: 24905070]

98. Cunningham S, Wake D, Waller A, Morris A. Definitions of eHealth. In: Gaddi A, Capella F, Manca M, editors. eHealth, Care and Quality of Life: Forewords by Sergio Bertolucci and Gianfranco Gensini. New York and Heidelberg: Springer; 2014:15-30.

99. Gaddi A, Capello F. The debate over eHealth. In: Gaddi A, Capello F, Manca M, editors. eHealth, Care and Quality of Life. New York and Heidelberg: Springer; 2014:1-13.

100. Greenhalgh T, Wherton J, Papoutsi C, Lynch J, Hughes G, A'Court C, et al. Beyond adoption: a new framework for theorizing and evaluating nonadoption, abandonment, and challenges to the scale-up, spread, and sustainability of health and care technologies. J Med Internet Res 2017 Nov 1;19(11):e367 [FREE Full text] [doi: 10.2196/jmir.8775] [Medline: 29092808]

101. Arnhold M, Quade M, Kirch W. Mobile applications for diabetics: a systematic review and expert-based usability evaluation considering the special requirements of diabetes patients age 50 years or older. J Med Internet Res 2014 Apr 9;16(4):e104 [FREE Full text] [doi: 10.2196/jmir.2968] [Medline: 24718852]

102. Parker SJ, Jessel S, Richardson JE, Reid MC. Older adults are mobile too!Identifying the barriers and facilitators to older adults' use of mHealth for pain management. BMC Geriatr 2013 May 6;13:43 [FREE Full text] [doi: 10.1186/1471-2318-13-43] [Medline: 23647949]

103. Stellefson M, Chaney B, Barry AE, Chavarria E, Tennant B, Walsh-Childers K, et al. Web 2.0 chronic disease self-management for older adults: a systematic review. J Med Internet Res 2013 Feb 14;15(2):e35 [FREE Full text] [doi: 10.2196/jmir.2439] [Medline: 23410671]

104. Chang BL, Bakken S, Brown SS, Houston TK, Kreps GL, Kukafka R, et al. Bridging the digital divide: reaching vulnerable populations. J Am Med Inform Assoc 2004;11(6):448-457 [FREE Full text] [doi: 10.1197/jamia.M1535] [Medline: 15299002]

105. Showell C, Turner P. The PLU problem: are we designing personal ehealth for people like us? Stud Health Technol Inform 2013;183:276-280. [Medline: 23388298]

106. Jonkman NH, van Schooten KS, Maier AB, Pijnappels M. eHealth interventions to promote objectively measured physical activity in community-dwelling older people. Maturitas 2018 Jul;113:32-39. [doi: 10.1016/j.maturitas.2018.04.010] [Medline: 29903646]

107. Muellmann S, Forberger S, Möllers T, Bröring E, Zeeb H, Pischke CR. Effectiveness of eHealth interventions for the promotion of physical activity in older adults: a systematic review. Prev Med 2018 Mar;108:93-110. [doi:

10.1016/j.ypmed.2017.12.026] [Medline: 29289643]

108. Barretto AI, Bingham CR, Goh KN, Shope JT. Developing a web-based health promotion intervention: a case study from a brief motivational alcohol program. Health Promot Pract 2011 Mar;12(2):193-201. [doi: 10.1177/1524839909353740] [Medline: 21051327]

109. Thomas J, Barraket J, Wilson C, Cook K, Louie Y. Measuring Australia's Digital Divide: The Australian Digital Inclusion Index 2018. Australian Digital Inclusion Index. 2018. URL: https://digitalinclusionindex.org.au/wp-content/uploads/2018/ 08/Australian-digital-inclusion-index-2018.pdf [accessed 2020-06-30]

110. Monkman H, Kushniruk AW. The consumer health information system adoption model. Stud Health Technol Inform 2015;218:26-31. [Medline: 26262522]
Abbreviations
eHLF: eHealth Literacy Framework
eHLQ: eHealth Literacy Questionnaire
ICT: information and communications technology
RCT: randomized controlled trial
UDHHS: US Department of Health and Human Services
WHO: World Health Organization 
Edited by G Eysenbach; submitted 28.02.20; peer-reviewed by L Griebel, H Monkman, E Brainin; comments to author 18.04.20; revised version received 30.04.20; accepted 03.06.20; published 13.08.20

Please cite as:

Cheng C, Beauchamp A, Elsworth GR, Osborne RH

Applying the Electronic Health Literacy Lens: Systematic Review of Electronic Health Interventions Targeted at Socially Disadvantaged Groups

J Med Internet Res 2020;22(8):e18476

URL: http://www.jmir.org/2020/8/e18476/

doi: $\underline{10.2196 / 18476}$

PMID: 32788144

(C) Christina Cheng, Alison Beauchamp, Gerald R Elsworth, Richard H Osborne. Originally published in the Journal of Medical Internet Research (http://www.jmir.org), 13.08.2020. This is an open-access article distributed under the terms of the Creative Commons Attribution License (https://creativecommons.org/licenses/by/4.0/), which permits unrestricted use, distribution, and reproduction in any medium, provided the original work, first published in the Journal of Medical Internet Research, is properly cited. The complete bibliographic information, a link to the original publication on http://www.jmir.org/, as well as this copyright and license information must be included. 